\title{
La vespa e il suo nido nelle Lingue romanze.
}

Assolvo qui in parte la promessa ch' io ebbi a fare or non è molto, occupandomi dei nomi dell' ape e dell' alveare ${ }^{1}$; dico in parte, perchè non mi è dato per ora di studiare $\mathrm{i}$ nomi che ho ráccolto del calabrone. $\mathrm{Di}$ questo insetto si hanno delle varietà molto dissimili tra loro che il popolo spesso distingue e designa con nomi diversi, senza però rendersi conto, com'è naturale, dei loro corrispondenti scientifici. Per questo la ricerca è difficile e spesso ci lascia incerti sul valore delle singole voci: infatti per

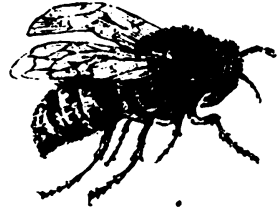

Fig. I. Vespa crabro.

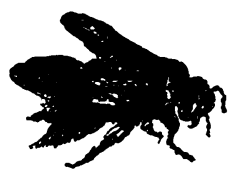

Fig. II. Xilocopa violacea.

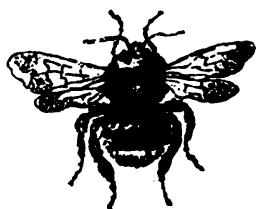

Fig. III. Bormbus terrestris.

esempio l' it. lett. 'calabrone' indica tanto la VRSPA CRABRo (v. Fig. I), 2 quanto la Xilocupa viol.acka (v. Fig. II), quanto il bombus nelle sue numerose varieta (TRKRESTRIS [v. Fig. III], HORTORUM, MUSCORUM, SYLVARUM ecc.); il francese lett. distingue tra 'frelon' (vRSPA CRABRO) e 'bourdon' (BOMBUS TRKRRSTKIS), ma non ha un nome particolare per la XILOCOPA violaCra. Bisognerebbe poter attingere in ogni caso direttamente dalle fonti orali e mostrare a queste le varie specie d' insetti che c' interessano, ma ognun vede quanto sia complicata per non dire impossibile la ricerca condotta in questo modo. Quindi mi limito a dare i nomi della 'vespa' e del 'vespain',

1 'L' ape e l'alveare nelle lingue romanse', Pisa, Mariotti, 1919. Cito questo mio opuscolo con la sigla $A p e$ e Alv.; le fonti di cui mi son valso per questo articolo sono pressappoco le stesse che mi servirono per l' articolo precedente. Ricordo pertanto che col nome dell' autore designo i vari dizionari dialettali da cui ho attinto, e che accompagno con le sigle Voc. Sv. It., Atl. e Atl.C. i materiali derivati rispettivamente dallo schedario per il Vocabolario della Svizzera Italizna, dall' Atlas linguistique de la France e da quello de la Corse. La sigla com. indica che la voce mi fu comunicata dai niei cortesi informatori; a questi si aggiunge ora il Dott. Adriano Garbini di Verona $e$ il Prof. Dr. K. Jaberg dell' Università di Berna i quali ringrazio di cuore.

2 Debbo le illustrazioni del mio articolo alla cortesia della Signa. Prof. Della Chiostra e dell' Ing. Giulio Buoncristiani; all' una e all' altro vadano le mie grazir vivissime. 
augurandomi di potere col tempo, non solu esaurire il compito che mi ero prefisso, ma anche tentar di risolvere i problemi biologici e genealogici che riguardano i nomi di cui mi occupo. I materiali che sono oggi a mia disposizione non mi consentono di farlo con quella sicurezza che vorrei; tali studi infatti, quando si voglia evitar d' innalzare un edificio vacillante perchè fondato su di un processo d' induzioni e deduzioni arbitrarie, richiedono una larga base di dati esattissimi la quale per ora mi manca.

\section{La vespa (VESPA).}

La vespa, come il calabrone, comprende parecchie varietà (VESPA vUlgaris [v. Fig. IV], GRRMANICA [v. Fig. V] RUPA, SYI.VESTRIS, polistes Gallica [v. Fig. VI], ecc.), ma queste, tanto per la forma, quanto per le dimensioni e per il loro modo di vivere, non differiscono molto l' una dall' altra, sicché soltanto l' entomologo sa fare le debite distinzioni; il popolo confonde tra loro le specie e le

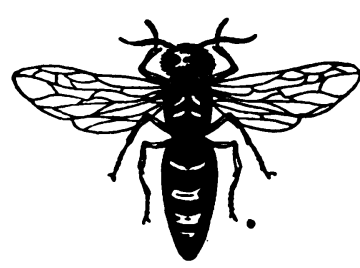

Fig. IV.

Vespa vulgaris.

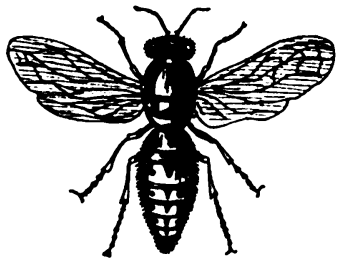

Fig. V.

Vespa germanica.

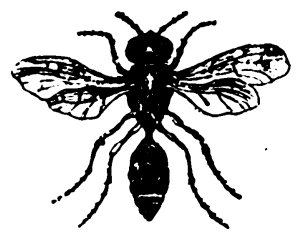

Fig. VI.

Polistes gallica.

indica tutte con un sol nome. Anche per la vespa si può ripetere quello che osservai già a proposito dell' ape: le denominazioni con cui si designa il nostro insetto, pur tanto caratteristico e diffuso, non rispecchiano in generale le sue peculiarità più evidenti (la conformazione del corpo, le abitudini di vita, la ferocia, ecc.), giacché il nome latino si è diffuso e mantenuto saldamente quasi dappertutto.

Sono tuttavia notevoli nei dialetti sardi, nel lorenese e nel vallone, alcuni casi di fusione di VESPA + APIS ai quali si hanno dei bei riscontri nelle voci che indicano l' ape e che già misi in rilievo ;' come pure di contro ai nomi dell' 'ape' derivati da quelli dell' 'alveare', 2 abbiamo, in alcuni dialetti della Francia, quelli della 'vespa' derivati dal 'vespaio'. Mentre nelle parlate d' Italia ha una certa diffusione 'vespa' usato a indicare l' 'ape', 3 il caso contrario ('ape' per 'vespa') si riscontra isolato a Levanto, a Bari e in qualche dialetto provenzale. I nomi della vespa derivati da quelli di altri insetti (come 'fuco', 'calabrone', 'mosca') sono del

1 V. Ape e Alv., pag. 28.

2 lb., pp. 34-35.

8 Ib., pag. 28. 
rumeno e di poche parlate della Francia, più che altro della Provenza, laddove, specialmente nell' emiliano, hanno una certa diffusione $i$ derivati di AsILUS che, in molta parte del territorio romanzo, indica il 'tafano'. Soltanto in due località (a Novellara e a Parma), ho trovato due nomi che accennano alla forma della vespa: navonzin e timonzen riavvicinano certo, nell' immaginazione del popolo, l' ape alla nave, ma mi par difficile stabilire in modo sicuro se il riaccostamento delle due idee avviene direttamente, oppure per mezzo di un intermediario. 'Navicella' è diffusissimo nei dialetti italiani per indicare la 'spola' (cfr. anche il fr. 'navette') e la forma allungata, rigonfia nel mezzo e sottile alle estremità della vespa richiama tanto quella dello scafo di una nave, quanto quella della spola. A chi osservi come la vespa suole interrompere bruscamente il suo volo per battere contro un ostacolo e pungere e quindi tornare rapidamente indietro, quasi rifacendo in senso contrario il cammino già fatto, si desta subito

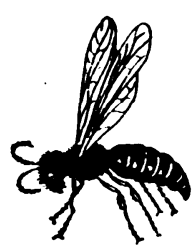

Fig. VII. l' immagine della spola che scorre con moto uniforme da un capo all' altro della tela; però il trovare il nome della vespa adoperato a indicare una specie di battello (v. p. $30+$ n. 7) ci fa pensare che il nostro insetto abbia potuto direttamente suscitare l' immagine di una piccola nave, di cui le vele potrebbero essere raffigurate nelle ali in posizione di volo (v. Fig. VII).

\section{VĚSPA :}

\section{A.}

I. rum. yaspă -; Rabins la viäs̆pa com., Lenz las r'ǵašpras ib., Erto, Fassa béspa, Vigo bešpp, Buchenstein, Ampezzo vě̌pa, Badia S. Leonardo oéšpa, S. Vigilio éšpa, friul. jespe (ĝ̀spe), Muggia biespa -; Breno ra vespe Voc. Sv. Il., Mergoscia er vespa ib., Vanzone (Ossola) víspa ib., Vogogna (Ossola), Gandria, Lamone, Caviano, Cugnasco "espa ib., Crealla (Canobbio), Rovio, Viggiù véspa ib., Palagnedra-Moneto, Loco, Auressio, Minusio, Brissago, S. Abbondio, Peccia, Broglio, Cavergno Bignasco, S. Antonio (V. Morobbia), Dalpe, Viganello, Torricella, Stabio, Balerna, Riva S. Vitale la respo ib., Crana la vespaa ib., Russo, Linescio, Menzonio, Carasso, Faido, Giornico la vešpa ib., Cavigliano, Campo V. Maggia, Valsolda resspa ib., Certenago, Roveredo (Mesolcina) la vešpa com., 1 Soazza (Mesolcina) la vešpa (plur. -on) ib., Ascona la vešpa Voc. Sv. It., Bedigliora (Malcantone) vẹspa (plur. vešsp) com., Roveredo (V. Capriasca) véšpa (plur. véšp̧) ib., Mesocco, Bedretto vešspa ib., Golino la ves.spa Voc. Sv. It., Locarno vispa ib., Maggia, Rossura, Pura vespa ib., Gerra Gamb. vešpa ib., Osco, Rivera, Malnate (Varese) vęspa ib., Soragno ra vešpa ib., Valvestino bešpo ${ }^{2}$ Battisti in Sitzungsb.

1 Devo queste e numerose altre voci lad., prov., franco-prov. e piem. alla squisita cortesia del Prof. Dr. K. Jaherg di Berna.

8 Accanto a besplo. 
Kais. Akad. CLXXIV, 47, mil. vespa Banf, bergam. besba, Tiraboschi, liresc. crem. vespa Melchiori 315, Fumagalli 377; pav. vespa Manfredi, Corteolona, Sannazzaro de' Burgondi, Canneto Pavese, Mirabello, Mezzanino Po, Ferrera Erbognone, Bressana Bottarone, S. Cristina, Albuzzano, Pieve Albignola, Tromello, Valle Salimbene, S. Leonardo, Gattinara, Stradella, Cà della Terra, Travacò Siccomario, Torre del Mangano, Pancarana lo vespo (plur. $i$ vęsp) com., Casteggio, Battuda, Castel Rotto, Pinarolo Po, Montepagano, Sommo Lomellina, Spessa, Villanterio, Mirabello la vespa (plur. $i$ vęsp) ib., Voghera, Vigevano ra vęspa ib., Piacenza, Forlì vespa; piem. vespa, valses. vespa, Viverone, Bresso, Traversella, Rueglio, Feletto, Perosa Argentina, Casale Monf., Pozzolo (Aless.) vespa com., Torino, Chieri, Cuneo vespa ib., Locana vespa ib., Ivrea uespa, Tortona vęspo ib.; Genova e prov., Chiavari, Monterosso al mare vespa ib. - Sard.: Lanusei ĝespa, Tempio vespa ib., Calangianus espa ib., Sassari vilifa Atl. C. 789 - venez. arc. bespa Parodi in Ro. XXVII, 222, contado bell. bespa ib.,, Malcesine (Verona) bespa com., Venezia, Verona, Treviso, Trentino vespa ib., Udine, Cividale gespe (plur. gespis) ib., Chiesanuova guèspa ib., - Corso: vespa, bespa, wespa Att. C. 789. - Aquila, Pescocostanzo, Chieti, Lanciano vespa com., Sannio vespa Nittoli, Sora uęspa Merlo, Fon. del. dial. di Sora in Ann. Univ. Tosc. IV, 185; S. Severo (Foggia), Ugento (Lecce) vespa com., Andria vespe, Lecce espa; Melfi (Potenza) vespa; calabr., Reggio Cal.; Mandanici, Graniti, Girgenti, Catania, Trapani vespa com., Piazza Armerina vespa Roccella, Geraci siculo vęšpa com. - Roma vespe com., Velletri, Tivoli, Canino, Corneto Tarquinia vespa ib., Castro dei Volsci vespa, Subiaco éspa; Perugia, Pozzuolo, Spoleto, Sanpatucchio vespa com. - Pisa, Chianni, Volterra, S. Vivaldo, Piancastagnaio, Gaiole, Arezzo, Seravezza vespa ib. -; Groscavallo, Mondrone vẹspa ib., Ceresole Reale la véfa ib., Ayas vepe Atl. 672, Courmayeur, Aoste, Chatillon, Champorcher, Thônes (H.-Savoie) vépa ib., Oulx, Lanslebourg (Savoie) ğépo ib., Maïsette gépo ib., Bobi ğéspa ib.; Genève ğepa com., S. Maurice gépa (antiq. úépa) Atl. 672, Gingins ĝ́pa ib., Neuchâtel vousp com., Nendaz, Châble, Bourg-S. Pierre, Gruyères, Echallens y Belfaux, Billens "uipa ib, Vevey dyepa ib, Le Brassus gifpa ib., Fribourg vouepa (vouépa) com., Le Pont vuépa Atl. 672," Lens, Evolène ueffa ib., Vissoye uneif $a$ ib.; Pringy (H.-Savoie), Plaisia (Jura), Lent,

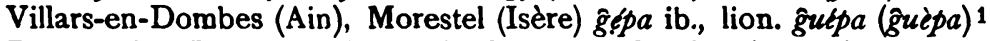
Puitspelu, La Biolle (Savoie), Gilley, La Rivière (Doubs), Morbier (Jura), Surjoux, Torcieu (Ain), H.-Savoie ${ }^{2}$ uépa Atl. 672, Avoudrey (Doubs) uep ib., Montain (Jura) uep ib., Gatey (Jura) gep ib., Mouchard, S. Amour (Jura) gépo ib., Vaux-lez-Molinges (Jura), Brion (Ain) vuépa ib., Ney (Jura) dyepa ib., Replonges (Ain) ġiépo ib., Savoie ${ }^{3}$

1 Accanto a tona.

2 A Pringy dicono $\hat{g} e ̣ p a$, a Thônes, vépa.

- A La Biolle dicono uspa, a Lanslebourg, ģspa. 
véppa ib., S. Priest, Le Bourg-d' Oisans (Isère) geépi ib., Sassenage

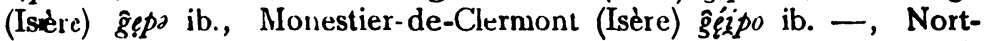
Leulinghem, Bainchtun' (Pas-de-C.), Fort-Mardyck, Glageon (Nord), Lanchères, Bouttencourt, Breilly, Oneux (Somme), Quincampoix, Achy, Plainval, Allonne, S.-Martin-Longueau (Oise) uǵp Atl. 672, Bois-Jean (Pas-de-C.), Le Châtelet (Arden.), Valleroy, Jaulny, Mailly, Moncel-sur-Seille (Meurthe et-M.) uép ib., Courcelles-Chaussy, Verdenal wep' Horning in Fr. St. V, 122 , Centre gêpe Rolland III, 27 I, Thieulain, Haraucourt (Arden.), Talmontiers (Oise), Barc, Breux (Eure), Suippes, Huiron (Marne), Thonne-les-Prés, Eix (Meuse), Thil, Sexey-aux-Bois (Meurthe-et-M.), Champlitte 2 (H.-Saone), Lamancine, Coiffy-le-Haut, Cour-l'évêque (H.-Marne), Aube, ${ }^{3}$ Molinons, Maligny, Cruzy-le-Châtel (Yonne), Eure-et-L., Orne, 4 Villerville, Beuvron-en-Auge, La Chapelle-Yvon (Calvados), Créances, Ponts (Manche), Uzel, Le Loscouet (Cotes-du-N.), Ille-et-V. 5, L'Ile d'Yeu, Avrillé (Mainc-et-L.), Indre-et-L., ${ }^{6}$ Loir-et-C. ${ }^{7}$, Gissey-sous-Flavigny, Martrois, S. Martin-de-la-Mer, Morey, La Rochepot, Mirabeau-surBèze (Cote-d'Or), Nièvre, ${ }^{8}$ Culan (Cher), La Vernelle, Chaillac (Indre), Saone-et-L. 9 , Allier ${ }^{10}$ ğep Atl. 672, Grandpré (Arden.), S. Pol-surTernoise 11 (Pas-de-C.), Martigny-en-Thiérache, Dizy-le-Gros, Suzy, Chartèves, Sains-Richaumont 12 (Aisne), S. Christophe-sur-Condé 13 (Eure), Le Plessis-Piquet (Seine), Courtisols 14 (Marne), Arrancy (Meuse), Crépey (Meurthe-et-M.), Poilly (Loiret), S. Jean-la-Poterie (Morbihan), Boëssé-le-Sec, S. Pierre-du-Lorouër (Sarthe), Avot-leGrand, Esbarres (Cote-d'Or), Guesnes, Dissay (Vienne), S. Claud (Charente) $\hat{g}_{\ell p}$ ib., S. Lager (Rhone) gepa ib., Chazelles (Charente) geppo ib., Vélu (Pas-de-(:), Vrély, Jumel (Somme) vöp ib., Marne roepe Rolland III, 270, Bussang vep' Horning in Fr. St.V, 122, Bayeux, picard. vepe, vepe Rolland III, 270, Vagney, Vosges vespe ib., Isbergues, 15 Baincthun, 16 Torcy, Teneur, Pierremont, Ramecourt, Verquigneul, Ligny-S. Flochel, Manin, Roeux (Pas-de-C.), Bruille-S. Amand (Nord), Vermand (Aisne), Bussy (Oise), Auderville, Sainte Geneviève (Manche) vgp Atl. 672, Pont-Hébert (Manche), Le Val-d'Ajol, Ramonchamp (Vosges) vep ib., vallone e loren. wes

1 Accanto a vep.

- Esiste anche una forma ant. vepr.

A S. Etienne dicono iep, a Auxon, ğiep.

- A Avoines dicono $\widehat{g} \dot{i} e p$.

5 A La Gouesnière dicono ĝ eip.

- A. S. Benoit si dice $\widehat{g} \dot{k} p$.

7 A S. Ouen dicono geppr.

- A Oudan si dice dyepr.

- A Beaubery dicono ptijet ĝ epy, a Davayé, ĝ̀iep.

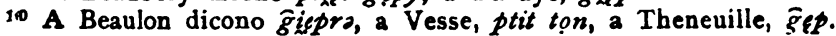

11 Dicono anche vep-v\&p.

12 Dicono anche mus puedron.

13 Dicono anche vrep.

1. Dicono anche yöp voce antiq.

15 Dicono anche $\hat{g} a n k u$.

is Dicono anche uep. 
Horning in ZRPh. XVIII, 230, Bliensbach, Gérardmer, Fréland, St. Marguerite wes' Horning in Fr. St. V, 122, La Poutroye wies' ib., Belmont, St. Blaise la Roche, Fouday wes' ib., Mons uesse, weche, wesse Rolland III, 27I, Waremme, Beaufays, Dolhain, Mesvin, Gordarville, Sclayn, Thirimont, Hanzinne, Bonnal-lez-Durbuy, Vielsalm wes Atl. 672, Lessines, Grupont, S. Pierre wasp ${ }^{1}$ ib., Wavre, Anseremme, Gedinne, Aublain, uূsp ${ }^{1}$ ib., Bouillon wáš ib., Chiny, Vance, Surmonne, Charbogne, Haraucourt 2 (Arden.) uap ib., Franche-Comté, Lorraine, Champagne vìtche, vôsse, vouésse, ouéche, oudsse Rolland XIII, 41, Haybes (Arden.) was Atl. 672, Beaubec-la-Rosière ${ }^{3}$ (Seine-Inf.), Blangy-sous-Poix 4 (Somme), S. Pierre-Port, Noyal-Muzillac, Loyat (Morbihan), Noirmoutier, S. Benoît (Indre-et-L.), Sillards (Vienne), Voultegon, Chef-Boutonne (D.-Sèvres), Chassors (Charente), Davayé (Saone-et-L.) ġępp ib., Linthes (Marne), 5 Auxon (Aube), Moutiers (Yonne), Avoines (Orne), Quetteville (Manche), Plouvara, Plévenon, Noyal (Cotes-du-N.), Crédin, Plumelec (Morbihan), Mayenne, Domfront-en-Champagne, Villaines-sous-Malicorne (Sarthe), Loire-Inf. ${ }^{6}$, Bouzillé, Chazé-surArgos, Chemillé, Soulanger, Bocé (Maine-et-L.), Berry-Bouy, Bué, Bruère (Cher), Chermignac, Guitinières (Charente-Inf.), Yviers (Charente), Cours (Rhone) g̀iep ib., Bully (Rhone) gigepa ib., Varennes (Somme) veöp ib., Jaulzy (Oise) vu€p ib., Ormoy-la-Rivière ${ }^{7}$ (S.-etOise) djęp ib., Longueville, Chenou (S. -et-Marne), S. Germain-lePrinçay, Charzais, Saligny (Vendée) $d y$ ep ib., La Celle-S. Cyr (Yonne), La Ferrière-Harang (Calvados), S. Clément (Manche), Flavigny (Cher), Pouligny-S. Pierre, Argenton (Indre), Oroux, Le Breuil-Bernard (D.-Sèvres), Clavette (Charente-Inf.), Angeduc (Charente) dyẹp ib., Gommecourt (S.-et-Oise) gip ib., Reims gouêpe Rolland III, 270, Igney (Meurthe-et-M.) uöp Atl. 672, Courtisols (Marne) uöp (voce ant.) 8 ib., La Petite-Raon (Vosges) uip ib., S. Etienne (Aube), S. Genou, Neuvy-Pailloux (Indre) isp ib., Saintonge yipe Rolland III, 27 I, Jort, Feuguerolles-sur-Orne (Calvados), Trévron (Cotesdu-N.) g gip Atl. 672, La Gouesnière (Ille-et-V.) ġsip ib., Vienne jaipe Rolland XIII, 27 I, Blanzay (Vienne), Varaize (Charente-Inf.) ğiepe Att. 672, Ligugé, Millac (Vienne), Sainte-Marie, S. Groux (Charente) gąp ib., Prissé, Echiré (D.-Sèvres) diạp ib., Pamproux (D.-Sèvres), Triaize (Vendée) dịasp ib., Givraud (Vendée) djip ib., Talmont (Vendée) dịip ib., La Verrie (Vendée) ğ $a_{e} p$ ib., Cabariot La Tremblade (Charente-Inf.) hį̣p ib., La Cotinière hį̣p ib., S. Symphorien-sur-Coise (Rhone) geppi ib. - prov. vespa ${ }^{9}$ Raynouard

1 Vi. Gilliéron, Gén., pag. 140.

Dicono anche $\hat{g} e p$.

8 Dicono anche vepr.

- Dicono anche uep.

5 Dicono anche vkep (voce ant.).

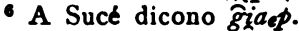

7 Dicono anche mus diet.

- Dicono più comunemente $\hat{g} \in p$.

- Accanto a vespo e guespo Azaís II, 385 e III, 684. 
V, 527, Allanche (Cantal), Puget-Théniers (B.-Alpes), S. Sauveur, Fontan, Menton ${ }^{1}$ (Alpes-M.) vespa Atl. 672, Vernante, Limone vęspa com., Var ręspo Mistral, Trésor II, 107, Coussac-Bonneval (H.-Vienne)

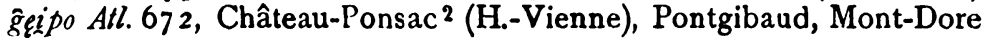
(Puy-de-D.), S. Savin, Abzac (Gironde) ğep ib., Eymoutiers (H.-Vienne) ğąqpi ib., Limoges (H.-Vienne) ğáepo ib., S. Quentin (Creuse) dịöpa ib., Lavaufranche (Creuse), Langogne (Lozère) gępo ib., Biarritz (B.-Pyren.) gepp ib., Cressat (Creuse) ġispo ib., S. Dizier (Creuse) gecipo ib., S. Eloy-les-Mines, Thiers, Ambert (Puy-de-D.), Riotord (H.-Loire), Merlines (Corrèze), Vion (Ardèche), Castillon (Ariège) gẹpo ib., Pral (Vald.) geppo com., Ennezat, Monton (Puy-de-D.), Seilhac (Corrèze), Laguiole (Aveyron), S. Nazaire-en-Royans, Chabeuil (Drome), Martigues (B.-du-Rhone), Agde (Hérault) ĝ́po Atl. 672, S. GermainLembron (Puy-de-D.), S. Chely-d'Apcher (Lozère), Aramont, Fourques (Gard), Vaucluse, Moissac (Tarn-et-G.), Sigean, Rivel, Axat (Aude) ğespo ib., Vélines (Dordogne), Seyches, Sainte-Livrade, Aiguillon (Lot-et-G.), Villefort (Lozère), Les Ollières, Burzet, Vogüé (Ardèche), Die, Marsanne, Luc-en-Diois, Nyons (Drome), Aiguilles, Guillestre, Veynes (H.-Alpes), Barjac (Gard), Courthézon, Villelaure (Vaucluse), S. Etienne-les-Orgues, Mezel (B.-Alpes), S. Maximin (Var), Eyguières, Gardanne (B.-du-Rhône), Nissan (Hérault), Saverdun (Ariège), Ladern (Aude) gespo ib., Sampeyre gespq com., Gers, Aix, Queyras ĝuespo Rolland III, 270, Delfinato, guespo (gueipo) ${ }^{3}$ Mistral, Trésor II, 107, Sail-sous-Couzan (Loire), Paulhan, Frontignan, (Hérault) gẹpa Atl. 672, S. Bonnet-les-Château (Loire), Paulhaguet (H.-Loire), Nassiac (Cantal), Caveirac (Gard) ġepa ib., Chamalières (H.-Loire) gespa ib., Solignac-sur-Loire (H.-Loire), S. Agrève (Ardèche), S. Firmin, Chorges, Orpierre (H.-Alpes), Sault (Vaucluse), Châteaufort, Barcelonnette (B.-Alpes), Lodève (Hérault) ĝ̨spa ib., Angrogna gespa com., Bobbio gespa ib., Entracque gespä ib., Crissolo ğespa, Bort (Cantal) gapo Atl. 672, Pleaux (Cantal), Villefranchede-Belvès (Dordogne), Souillac (Lot), Hyères (Var) gęhpo ib., Vicsur-Cère, S. Mamet (Cantal), Tournon-d' Agenais (Lot-et-G.), Gramat, Gourdon, Figeac, Promilhanes (Lot), Conques, Rieupeyroux (Aveyr), bęho ib., Meymac (Corrèze) gęhpa ib., La Roche-Canillac (Corrèze) "ghp. ib., Beaulieu (Corrèze) "'gpo ib., Larche (Corrèze), Issigeac, Le Bugue (Dordogne) gesspo ib., Andraut (Gironde) gáęa ib., S. Vivien, Lacanau, Targon (Gironde) ĝgespi ib., Cissac, Pessac, S. Côme (Gironde), Houeillès (Lot-ct-G.), La Javie (B.-Alpes) ğgspo ib., Pierrelatte (Drome) gespso ib., linguad. e guasc. bespo Mistral Trésor II, 107, Séverac-le-Château, Belmont (Aveyr.), Valderiès, Gaillac, Brousse (Tarn), Montpezat, Beaumont (Tarn-et-G.), Montasiruc, Martres-Tolosane (H. Garonne) bespo Atl. 672, Layrac, Mézin (Lotet-G.), Cahors (Lot), Espalion, Calmont, S. Rome-de-Tarn, Nant

1 V. anche Andrews in Ro. XVI, 55 I.

2 Dicono anche bek.

A Accanto a mousco-vairo, mouscho jalho. 
(Aveyr.), Marvéjols, Florac (Lozère), Lamalou-les-Bains (Hérault), Aussillon (Tarn), Laguépie, Grisolles (Tarn-et-G.), Gimont, Lectoure, Jegun, S. Martin, Lombez (Gers), Aureilhan, Gerde, Tramesaygues (H.-Pyrén.), Léguevin, Donneville, Revel, Carbonne (H.-Gar.), Le Mas-d' Azil, Crampagna, Mérens (Ariège), Fanjeaux, Tuchan (Aude) bespo ib, Tarn, Toulouse, Lauragais bèspo Rolla nd III, 270, Menếtierles-Bains (H.-Alpes) gáipa All. 672, Languedoc vespo Rolland III, 270, Sumène, Uzès, Alais (Gard), Castellane, Gréoux (B.-Alpes), Plan-du-Var, Le Cannet (Alpes-Mar.), Aups, Seillans, S. Tropez (Var) vespo Atl. 672, Le Luc (Var) vespo ib., Elva vespo com., Les Matelles (Hérault), Cauterets (H.-Pyrén.), Collioure, llle-sur-Têt, Arles-sur-Tech (Pyrén.-Or.) bespa Atl. 672, Auzat (Ariège) bespu ib., Rivesaltes (Pyrén.-Or.) bespa ib., Olette (Pyrén.-Or.) bespe ib. -; galiz. vespa Rolland III, 271 , portogh. bespa ${ }^{1}$, Parodi in $R o$. XXVII, 222.

S. Pol-sur-Ternoise (Pas-de-C.) vep-r'ep Atl. 672.

2. Forme di plur. passate al sing. (v. Meyer-Lübke, REW. 9272): rum. viéspe ${ }^{3}$, arom. yaspe, megl. gaspi Puşcariu -; Dissentis viášp G a rt ner, Raet. Gramm., §84, Mathon la vęašp com. -; Poschiavo la vęspi (plur. li vęspi) com., Bondo Promontogno la vesp Voc. Sv. It., Castasegna la vešp ib., Soglio la vešp ib., Stampa veisp ib., S. Nazzaro la vespe ib., Marzio vesp com. - Lari, Vicopisano, Lucca ${ }^{4}$ vespe.

3. Articolo conglutinato: Templeuve-en-Pévèle (Nord) nep 5 Atl. 672, Magny (Yonne) nepp ib., Morvan niçpe Rolland III, 271, Linselles (Nord) merp ${ }^{6}$ Att. 672.

catal. avespa Rolland XUI, 41 -; spagn. avispa ${ }^{7}$ e abispa Rolland XIII, $4 \mathrm{I}$, castigl. avispa Dicc. cast. 103 -; port. abespa. ${ }^{8}$

4. Epentesi: V. Gardena bešpia Gartner, Raet. Gramm. § 84, V. di Non bessp[j]a Battisti in Sitz. der Kais. Akad. der Wissensch. III, 142, trent. rustico bespia com.

soprasass. vespra A sc oli in A. G. It. I, I ro, soprasilv. viaspra ib., Flims la vęspra (plur. -as) com., Rhäziins, Reams, Bivio Stalla, Zuoz la vešpra ib., Samaden vespra Gartner, Raet. Gramm. § 84, Fex, S. Maria (V. di Monastero) la vešpra com., Schleins véspra, Gartner, Brigels la väšpra com., Compadials, Rueras (Tavetsch) la viášpra ib., Remûs, Stuls, Latsch (Bravugn) la vešspra ib., alto-engad. vespra Carisch 180 , bassoengad. veispra ib., Ardez la véy spra com. -; Leontica vispra ${ }^{9}$; Voc.Sv. It.; romagn. vèspra Morri, Ravenna, Alfonsine, Faenza, Forli, Cesena

1 Accantó a abespa.

2 Accanto a $\bar{g} \ell p$.

- Accanto a yaspa e vespa.

- Dicono anche vespre e vespra.

5 V. Gilliéron, Gen. pp. 205-213.

B Ib., p. 2 II.

7 Accanto a vispera, viespera, viespra.

8 Accanto a bespa.

- Accanto a vinispra (idiot.). 
rêsppre com., Terra del Sole, Castrocaro vespra ib. - Abruzz. vèspre Finamore 313, Civitella Alfedena vespra com., Teramo vespra ib., Città S. Angelo vešpra ib., Castelfrentano, Torricella Peligna vespra ib.; Agnone vespro ib. - Fano, Cagli, Macerata Feltria, Ancona, Fabriuno, Macerata, Fermo vespra ib. - Carrara, Codena, Miseglia, Torano, Fontia, Avenza, vespra ib., Sassalbo vęspra ib. - Lucca 1 e Val di Serchio vespra Nieri, 249 -; Jura Bernois vouitpr com., Courrendlin, Les Bois, Vuitteboeuf vuepr Atl. 672, Coeuve, S. Hippolyte, Torpes (Doubs) uepr ib., S. Braix, Péry uepr ib., Montbéliard vouiëpre Rolland III, 270, Le Landeron uipr Atl. 672, Joncherey, Clerval, Bouclans, Vuillafans (Doubs) vepr ib., Amange (Jura) ğepr ib. - L' île de Serk, Les Moitiers-d' Allonne, Fresville (Manche), Beaubec-la-Rosière, ${ }^{2}$ Bellengreville (Seine. Inf.), Montbozon, Autoreille (H.-Saone), Rougegoutte vepr ib., Humes (H.-Marne), Conflans-sur-Lanterne, Champlitte ${ }^{3}$ (H.-Saone) vepr ib., Plancherles-Mines, Pays de Bray, Champagne vêpre Rolland III, 270, Clécy (Calvad.), Echenoz-la-Méline, Gourgeon ${ }^{4}$ (H.-Saone), S. Ouen (Loiret-C.) gepr Atl. 672, Ronchamp, Fresne-S. Mamés (H.-Saone), Vanvey (Cote-d Or) ĝfpr ib., Verzenay (Marne) vuepr ib., Le Mesnil-sur-Oger vouêpre Rolland III, 270. Viller-la-Ville (H.-Saone) uepr Atl. 672, Velorcey (H.-Saône) ghetre Rolland XIII, 41 , Oudan (Nièvre) dyępr Atl. 672, Beaulon (Allier) ġiepro ib., La Garnache gìgpr ib. Velay vespro Mistral, Tresor II, 107, Vabre (Tarn) bespro Atl. 672, S. Pons (Hérault) bespro ib., Les Ternes (Cantal) behpro ib. -; spagn. viespra ${ }^{5}$ Meyer-Lübke, Gramm. Lang. Rom. I, \$ 181 .

Dualchi espru com.

Stampa la vẹyšpr (plur. lan véyšpr) - lucch. vespre Parodi in Ro. XXVII, 222.

Arcola, Spezia véspoa com., S. Lazzaro véspora ib., Sarzana véspura ib., Castelnuovo Magra vespoa ib. - nap. vespera D'A mbra 395, irpin. vespera Meyer-Lübke, REW. 9272 -; La Bressc (Vosges) vichpere Rolland III, 271 --; spagn. zispera e viespera ${ }^{\text {b }}$ Meyer-Lübke, Gramm. Lang. Rom. I, \$ I8I.

mant. vrespa Cherutini, parm. vrèspa 7 Pariset, regg. "rèspa Voc. reg. it. 1832, nod. vripa Maranesi, mirand. vrespa Meschieri, bulogn. vräspa Ungarelli, Imola vrespa com., romagn. vrespa ib., ferr. vrespa $\mathrm{Nannini,}$ Bondeno, Argenta vrespa com. - ven. brespa Boerio 70, Rovigo brespa o vrespa com., poles. brèspa Mazzucchi, a. pad. brespa Parodi in Ro. XXVII, 222, pad. brespa Patriarchi, veron. brespa 8 Angeli, Pescantina grespa com., Nogara,

1 Dicono anche vespre e vespe.

2 Dicono anche $\widehat{g}$ ijep.

8 Dicono anche $\widehat{g} e p$.

- Dicono anche ⿳⺈冖évolr.

- Accanto 2 avispa, vispera, viespera.

- Accanto a avispa e viespra.

- Accanto a timonsén.

- Accanto a grespa, vresta o vrespa com. 
Vicenza brespa ib., Treviso vrespa Ninni - bar. vrèspe Parodi in Ro. XXVII, 222 - ; Bourg-Beaudouin (Eure), S. Vaast-Dieppedalle; Yport, La Frenaye, Moulineaux, Bertrimont (Seine-Inf.) vrep Atl. 672 , $\mathrm{S}$. Christophe-sur-Condé (Eure) vrẹp ${ }^{1}$ ib. Bray, Cauchois vrêpe Rolland III, 270 - bord. grèspo Mistral, Trésor II, 107, La Testede-Buch, Hostens (Gironde), Parentis-en-Born, Mézos, Sabres, Luxey, Sarbazan, Pouillon (Landes), Aas, Sauveterre, Artix (B.-Pyrén.) bręspa Atl. 672, Grenade-sur-Adour, Hagetmau (Landes) bręhpo ib., Soustons (Landes) bröspo ib., Riscle, Eauze (Gers), Oloron, Lembeye (B.-Pyrén.), Lannemezan, Gavarnie, Sariac (H.-Pyrén.), S. Gaudens, Bagnères-de-Luchon (H.-Gar.) bręspo ib., béarn. brespo Mistral, Trésor II, 107 -; astur. briespa Meyer-Lübke, Gramm. Lang. Rom. I, § $18 \mathrm{I}$.

Lieu-S. Amand (Nord) verp' ${ }^{2}$ Atl. 672.

Matera (Basil.) la vresplee ${ }^{3}$ com.

Valvestino bešplo4, *brespro, *-splo Battisti in Silz. der Kais. Akad. der Wiss. CLXXIV, 47.

5. Assimilazione alla tonica (V. Salvioni in RILomb. XL, 728 ): Sementina le vešp $V_{0}$. Sv. It., Chironico lq vespe ib., Monte Carasso ir vèspe ib.

6. Derivati: Coulombs (S.-et-M.) ghepron Rolland XIII, 4I.

B.

1. 'Vespa gialla': La Trinité žaon vępr Atl. 672

2. 'La piccola vespa': Beaubery (Saone-et-L.) ptizt ĝepy Ail. 672.

3. a) veSPA + APIS 5: sardo camp. espi Spano, Cagliari, Pirri, Monserrato, Quartu S. Elena, Dolianova, Capoterra, Armungia, ecc. espi r. p.; log. espe Spano, Ozieri, Pozzomaggiore, Bosa, Mores, ecc. espe com., Bitti, Nuoro, Orani, Oniferi, Gavoi ĝ̨spe ib.; Siligo (Sassari) epe ib. 6 - arpin. vepa Salvioni in StR. VI, 6+ -; Malmédy, Bastogne ueps ${ }^{7}$ All. 672, llloud (H.-Marne), Gérardmer (Vosges) vuaz ib., Champ-le-Duc, Sainte-Marguerite, Arches (Vosges) uẹs ib., Romont, Fraize (Vosges), Einvaux (Meurthe-et-M.), La Poutroye, La Broque ų̧s ib., Demangevelle (H.-Saone), Courcelles-sur-Blaise, Poissons, Graffigny-Chemin (H.-Marne), Aubréville, Fresnes-au-Mont,

1 Accanto a ğ $\ell p$.

2 V. Gilliéron, Gén. 21 I.

3 Questa voce mí è comunicata dal Direttore della cattedra ambulante di agricoltura, ma il Festa (' $I l d l$. di Matera' in $Z R P h$ XXXVIII, 260) dà soltanto le forma väspo.

- Accanto a bespo.

5 Per i nomi dell' 'ape' derivati dallo stesso incrocio, v. il mio articolo Ape e Alv. pag. 28.

- Per queste forme sarde, v. Wagner, Lautl. süds. Mund., Halle a. S. 1907, $\$ 85$; ib. in $Z R P h .731-32$; Salvioni in RILomb. XLII, 855.

7 V. Gilliéron, Gén. 135 e 140. 
Ville-Issey, Sommelonne, Tréveray (Meuse), 1 Chatenois, Essegney, Racécourt, Attigny (Vosges) ${ }^{2}$, Belval (Marne) vọs ib., Ban de la Roche voisse Rolland III, 27 I.

b) APIS + VeSPA: Maurois (Nord) šugt 3 Atl. 672, Candas (Somme) exepp ib.

4. VESPA + 'mouchette' 4: La Longueville (Nord) uet Atl. 672, Augy (Aisne) węt ib., P.-de-C. ghétte Rolland XIL, 41.

5. Musca + vrspa. Sartrouville (Seine-et-O.), Marolles (Oise) $m u s ̌$ ğ $q p$ Atl. 672, Nibelle-S. Sauveur (Loiret) muš gięp ib, S.et-M., Loiret mouche-guespe, mouche d guêpe Rolland XIII, 4I, Bouilly (Loiret) mouche-guêpe ib. III, 270.5

II. 'Il vespaio' ':

Theys (Isère), Le Grand-Serre (Drome) ğeppì Atl. 672. Parrebbe che si dovesse muovere da un *vesPariu, almeno a giudicare dal confronto di alcune voci dell' Atl. [fumie Fumariv 618 , prẹmie

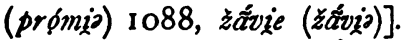

S. Broingt-les-Fossés (H.-Marne) véprgr m. All. 672. Forse da

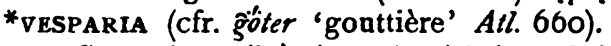

Charavines (Isère) ĝtppia Atl. 672, Saintes-Maries (B.-du-Rhone) véspio io ib. Potrebbero risultare da un *vespakiu rifatto, quanto alla vocal finale, su vesPa.

Les Voivres (Vosges) uípigt (Atl. 672) *vespariu + ottu.

III. Nomi derivati da insetti affini:

A. 'Ape', 'fuco', 'calabrone':

I. 'L' ape'?;

a) APIS: Lèvanto (Genova) ave com.

b) Derivati: Bari apone com.

La Ciotat (B.-du-Rhone) abijo 8 Atl. 672, Aigues Mortes (Gard) avojo ${ }^{8}$ ib., Monistrol-d' Allier (H.-Loire) bifia ${ }^{9}$ ib.

c) ' $L$ ' ape cattiva': linguad. abihasso, abelhasso in Mistral, Trisor I, 8, B.-du-Rhone marridei 11 abilio Rolland IIl, 27 I.

d) 'L' ape falsa': Legnago, Monteforte matonelu com. ${ }^{12}$

1 Il Rolland (III, 27 I) riporta per il dip. di Meuse la forma vôsse.

2 Il Rolland (ib.) dà anche ha forma voise.

3 V. Gilliéron, Gén., pag. 212.

- Ib., pag. 138.

- Cfr. mus abei 'ape' in Ape e Alv. pag. 32. pp. $34-35$.

- Cfr. i nomi dell' ape derivati da quelli dell' alveare in Ape $e$ Alz.

7 Cfr. i nomi dell' ape derivati da veSPa in Ape e Alv., p. 27.

- Queste forme sono pure usate a significare l'ape; v. Atl. I.

- Di contro a muš a midu; v. Atl. I.

$10 \mathrm{Cfr}$. lo spagn. abejonazo nel signif. di 'fuco'; v. Ape e Alv. p. 47.

11 Cfr. prov. marrit, ido (Mistral, Trésor JI, 284).

12 Cfr. i nomi del fuco derivati da mat (Ape e Alv. p. 49). A Verona, mattonsin o mattonsell dice anche 'calabrone'; v. Angell 39. 
2. 'Il fuco': Fiandra franc. malot' Rolland III, 27 I.

3. 'Il calabrone':

a) CRABRo: Gourgeon (H.-Saone) ĝtégolo 2 Atl. 672, Canton de Flavigny (Côte-d' Or) graivolon ${ }^{3}$ Rolland III, 27 I.

b) Burdo: Sainte-Anne burdõ Atl. 672.

c) *tabana: Clonas (Isère) tọna ${ }^{5}$ Atl. 672, Ain, Isère, Savoie tôna f., touna f. Rolland XIII, $4 \mathrm{I}$, lion. tona (pron. tona) ${ }^{6}$ s. f. Puitspelu, 40I, fores. tauna ib. - Allier tone f. Rolland XIII, 4 I, Rhône tôna f., touna f. ib. - Loire tôna f., touna f. ib., Néronde (Loire) tuina ${ }^{7}$ Atl. 672, Ambierle (Loire) tọn ${ }^{7}$ ib.

S. Jean-de-Bournay (Isère) șavã $8 \mathrm{~m}$. Atl. 672.

Vesse (Allier) ptit tọn 9 ib.

Qui vada anche il teneppe di Béthune (Pas-de-C.) ${ }^{10}$ che il Gilliéron 11 interpreta te-nep $=$ 'taon-nèfle' creatosi per evitare la confusione tra nep 'nespola' e nep 'vespa'.

d) *Carone: rum. gaaün Candréa-Hecht in Ro. XXXI, 3 I 2.12

e) Deriv. da 'foussou' = zappa: prov. foussoulou, foussalou Candréa-Hecht in Ro. XXXI, 312.13

f) Deriv. da 'fouca', 'foucha' = scavare, zappare: guasc. foucarou Candréa-Hecht loc. cit. 13

1 Cfr. malo = ape (Maurois) e $\mathrm{i}$ nomi del fuco derivati da Masculu; v. Ape e Alv. 33 e 50-52.

2 Cfr. i nomi francesi della 'vespa crabro' derivati da CRABRo Atl. 1172.

- La 'vespa crabro' è detta grôlon; v. Rolland III, 273.

- La stessa voce dice anche 'vespa crabro'; v. Atl. I572.

- Dice anche 'ape'; v. Ape e Alv. p. 33.

- Accanto a guepa.

- Queste stesse forme indicano anche il calabrone (vespa crabro), v. Atl. 1572; a Néronde, detta túna anche l' ape. v. Ape e Alv. pag. 33.

B Dice anche 'tafano' (v. Atl. 128I), laddove per designare il calabrone (vespa crabro) è usata la forma tọna (v. Atl. 1572). Nella cons. iniziale, sarà da vedere l'influsso di Cavare (cfr. säs $\delta$ 'chanson' Atl. 23I, sdrsi 'charger' ib. 239, ecc.).

Di contro a ton $=$ vespa crabro, v. Atl. 1572.

10 Rolland III, 27 I.

11 Gen. pag. 209.

12 L' etimo proposto dal Candréa-Hecht è messo in dubbio dallo Schuchardt (in $Z R P h . X X V I, 587-88$ ) il quale riconduce la voce rum. a CRABRo, nel che consente il Meyer-Lübke ( $R E W .2293)$, laddove il Puşcariu ( $R R P h$. XXVIII, 618) ritorna alla base proposta dal Candréa-Hecht.

${ }_{18} \mathrm{Cfr}$. le numerose forme dial. francesi simili a questa, le quali indicano il calabrone (vespa crabro), Atl. 1572. Il Meyer-Lübke (Misc. Ascoli, pag. 418) le riconnette con 'frelon' che fa derivare dal germ. borslo; ma l' idea di 'scavatore', 'insetto che fa i buchi' si ritrova oltre che nel rumeno ( $g$ aŭn), anche altrove: Macerata Feltria (Pesaro) bucarone com., Cagli (Pesaro) bugaron ib.; Auzances (Creuse) tal̨̧ro Atl. 1572 (V. anche Rolland XIII, 50) da TALIARE; Le Havre, Maze fouyon Rolland XIII, 49, Val d'A jol (Vosges) fouyb ib., Bessin feîyon, fiếyon Rolland III, 273, forse da *FODIONE. 
g) Tarn-et-G. fisso f., fissélou m. Rolland XIII, 4I (v. il prov. 'fisso' "pungiglione»). 1

h) Auzances (Creuse) burgọódo ${ }^{2}$ Atl. 672 (v. Meyer-Lübke, REW. 3597).

\section{B. 'La mosca'. 3}

1. 'La mosca gialla, screziata': delfin. mouscho-jalho, mousco vairot Mistral, Trésor II, 377.

2. 'La mosca dal pungiglione, che punge'.s Milly (Meuse) muš aริų̧ All. 672.

Tartas (Landes) mouskë caghilhë (Rolland XIII, 4I).

Sains-Richaumont (Aisne) muš puẽdrõn ${ }^{6}$ Atl. 672.

3. Ormoy-la-Rivière (Seine-et-O.) muš djêt Atl. 672, S. Ay (Loiret) $m u s ̌$ a jet ib. Secondo il Gilliéron (Gin. 80 e 138), sarebbe da intendersi 'mouche $d$ yet' cioè 'mouche de layettes' $=$ mouche de tiroirs ou coffrets (alvéoles); ma non vedo perché non possa interpretarsi invece 'mouche $d$ jet' ('mouche $a$ jet') $=$ la mosca dello sciame. .La tav. 1304 (tiroir) dell' Atl. registra per Ormoy-laRivière e S. Ay, tiruke, laddove lo sciame è detto što (v. tav. 482) $\mathrm{da}$ *JECrus secondo che richiede la mia interpretazione. Si dovrebbe perciò ammettere che 'mouche de get' indicasse anche l' ape prima che questa assumesse il nome che ha attualmente ('mouche a miel') e si avrebbe cosi una prova che la zona in cui dominò per alquanto tempo 'mosca dello sciame', 'sciame' fu molto più estesa dell' attuale (v. Gilliéron, Gen. pp. 4 I segg.). A 'mouche de get' $=$ 'mosca dello sciame' farebbe riscontro il je $d m u s$ ' $=$ sciame, che si ode a Vermand (Aisne) v. Atl. 482.7

4. MUSCA: Port-en-Bessin (Calvad.) muk Atl. 672.

1 A questa forma si riconnettono molte altre che dicono calabrone (vespa crabro) e che sono registrate dal Mistral (Trésor I, 1138), dal Azaïs (11, 246) e dall Atl. (tav. 1572, numri. 648, 658, 659, 741, 750, 752). Il Mistral (loc. cit.) proponc la base che mi sembra accettabile anche considerando che il calabrone d denominato dal pungiglione in varie localita del territorio romanzo; per es.: Velletri punciglione (accanto a vespone); Nièvre dard (R oll and XIII, 50), Woippy (Pay messin.) dâ (ib.), Ramecourt (Pas-de-C.) dar (Atl. 1572), certamente da DAROP (REW. 2479), cfr. dar 'aiguillon' a Ramecourt, Atl. 15.

2 Questa voce e certamente counessa con burgau = calabrone (vespa crabro) che et di molti dialetti della Francia e specialmente della Provenza (v. Atl. 1572 e Rolland III, 273). La terminazione della nostra forma accenna al suffisso '-aldo'.

- Cfr. i nomi dell' ape derivati da quello della mosca in Ape e Alv. pp. 28-33.

- Accanto a guespo (gueipo).

- Cfr. mohe d pespin, mohe al pepeye ecc. = ape. V. Ape e Alv. pag. $3 \mathrm{I}$.

- Accanto a $\hat{g} \notin p$.

1 Cfr. anche Plouvara, Noyal (Cotes-du-N.) jie do rus Atl. 482, Crédin, Plumelec (Morbihan) jö $d$ 'avęt ib., S. Jean-la-Poterie (Morbihan) jq d'an'̨t ib., Noyal-Muzillac (Morb.) je d'avęt ib. 
C. Asilus + Acoleus (v. Meyer-Lübke, $R E W$. 702): emil. asiol com., Borgoforte asiolo ib., mantov. asiöl ib., Carpi asiol ib. Trevenzuolo, Sorgà (Verona) asiöl ib.

D) Bas $^{1}$ (v. Meyer-Lübke, $R E W$. 1057): piacent. bsia 2 Foresti 96, parm. bsia ${ }^{3}$ com.

IV. Insetto in generale:

A. вомвуX 4: Charente bike Rolland XIII, 4I - Creuse bièco, obieco ib., H.-Vienne becco ib., S. Junien, Châlus (H.-Vienne), La TourBlanche, Excideuil (Dordogne) bęko Atl. 672, S. Pardoux-la-Rivière (Dordogne) bẹko ib., limousin beco Mistral Trésor II, 107, S. Pierrede-Chignac, Bourgnac (Dordogne) bẹko Atl.672, Château-Ponsac (H.-Vienne) bẹk $5 \mathrm{ib}$.

B. ted. 'Käfer': Le Tholy gébrate, gébrote, Rolland III, 27 I. v. Nigra in AGlt. XIV, 365 .

V. ' $L$ ' insetto di S. Martino':

mil. martinett ${ }^{6}$, bresc. martinel, Coccaglio martinel com.; pav. martinè, Vistarino, Retorbido, Alagna Lomellina al martiné (i martiné) com., Garlasco al marliné (i martiné) ib.

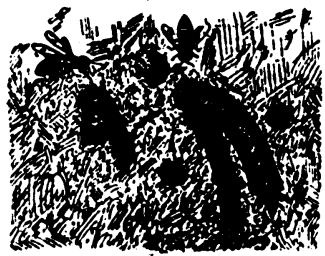

Fig. VIII.

Odynerus parietum

e il suo nido

VI. 'La navicella':?

A. NAvis: Novellara navonzin ${ }^{8}$ com.

B. TEMO: parm. timonzen. 9

VII. ' $L$ ' insetto muratore':

nizz. muradou s. m. Però qui si tratta della 'vespa muraiola' (Odynerus parietum; v. Fig. VIII).

VIII. Voci oscure:

A. Leontica vinispra ${ }^{10}$ (idiot.) Voc.Sv.It.

1 A milano, besci indica l'ape e il calabrone, v. Ape e $A l v$. pag. 33 e Cherubini, Susplem.

2 Accanto a vespa.

- Accanto a vrespa e timonsén.

- Cfr. Ape e Alv. pag. 33. Da questa base derivano anche alcuni nomi del calabrone: parm. bega mora (= xilocopa violacea) Malaspina I, 184, Forli biğarú (= vespa crabro) com. Alfonsine (Ravenna) bagaron ib. Per i nomi prov. v. Mistral, Trésor I, 286.

5 Accanto a gepp.

- Indica forse anche il calabrone (vespa crabro) perchè il Cappelletti (244) traduce 'frelr $n$ '. Del resto martinẹ o martinẹl significa calabrone anche a Pavia e nel territorio circostante. XIII, 47 .

7 Nel fr. dial, guêpe indica anche una specie di battello, v. Rolland

- Cfr. naonzelo $=$ calabrone, nel poles. (Mazzucchi I57) e naonséi o giaonzei detto pure di una specie di calabrone, a Rovigo (com.).

- Accanto a vrespa e bsia.

10 Accanto a vispra. 
B. Palmi (Umbria) lefina com.

C. Verona vresta. 1

D. Guernesey pimpernuche Rolland III, 27I. Il Rolland annota: "ainsi nommée à cause de ses bigarrures." $\mathrm{Ma}$ resta ad ogni modo oscura la desinenza.

E. La Chapelle (Meurthe-et-M.) obrgt Atl. 672.

F. Quarouble (Nord) vèrne Rolland XIII, 4 I.

G. Dun-le-Palleteau (Creuse) gẹep ĝepái Atl. 672.

H. Isbergues (Pas-de-C.) ga $a n k u^{2}$ Atl. 672.

\section{Il vespaio (NIDUS VESPARUM).}

Pochi insetti rivelano, nella costruzione del loro nido, un istinto artistico così sviluppato come la vespa. I nidi delle vespe sono formati, come quelli dell' ape, di tanti favi con le piccole aperture generalmente rivolte all' ingiù $e$ per lo più di materia vegetale che, opportunamente lavorata dall' insetto, prende l'aspetto di carta di

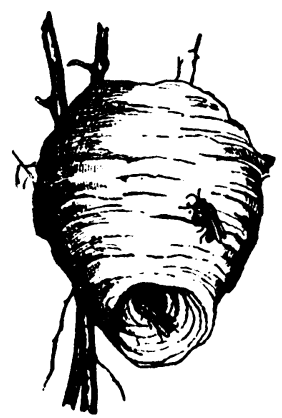

Fig. IX.

Nido di Vespa silvestris.

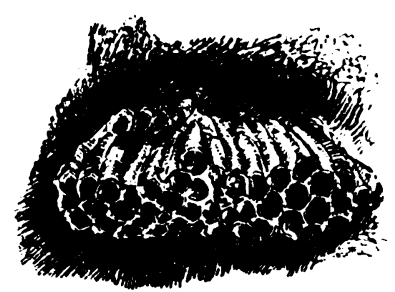

Fig. $X$.

Nido di Polistes gallica.

color bigio-scuro; però la materia di cui le vespe costruiscono la loro abitazione è spesso diversa nelle varie specie, alcune delle. quali adoperano anche della terra argillosa, oppure lo sterco di animali erbivori. Molto più varia è la forma che queste bizzarre costrunioni assumono; sì può dire che ogni specie costruisce.il suo nido con una forma caratteristica la quale varia anche nelle singole specie, secondo il luogo in cui l' insetto ha potuto stabilirsi. Cosi per esempio la vespa sylvestris costruisce il suo nido sopra gli alberi o i cespugli e gli dà una forma che a volte sembra un calice rovescialo, a volte, quando acquista proporzioni maggiori, un fiasco capovolto (v. Fig. IX); la vespa media fa la sua abitazione a forma di conchiglia, pressappoco come la polistes gallica che però suole formarla un pò più schiacciata, sicché non è difficile ravvisare nell' insieme delle cellette un piccolo pane rotondo (v. Fig. X). La vespa vulgaris, la

- Accanto a brespa, grespa o vrespa.

2 Accanto a v'ep.

Zeitschr. f, rom. Phil. XLIl. 
germanica e la rufa depongono i loro favi sotto terra, nelle gallerie scavate dalle talpe, ma non danno all' insieme una forma caratteristica, giacché debbono disporre le cellette secondo le dimensioni e la forma della cavità che trovano nel sottosuolo (v. Fig. XI).

Se può sorprendere la poca varietà dei nomi con cui il popolo designa la vespa, maggior sorpresa desta il trovare una grande uniformità anche nei nomi del vespaio che è tanto vario e caratteristico nelle sue forme. A queste accennano poche voci tra quelle

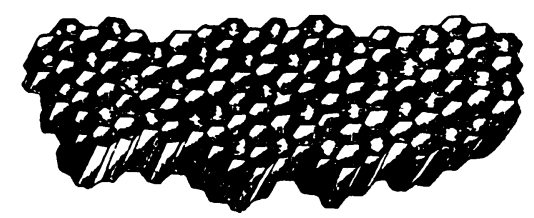

Fig. XI. Nido di Vespa vulgaris.

che ho raccolto, poichè, nella maggior parte delle lingue romanze, i nomi del vespaio sono derivati da quelli dell' insetto; dalla denominazione latina (NIDUS VESPARUM) muovono il sardo e qua e là soltanto alcuni fra $i$ dialetti ladini $e$ italiani, ma questi però accanto a 'nido delle vespe' hanno, in generale, anche le rispettive risoluzioni fonetiche di 'vespaio' che, come si vede, ha finito per trionfare quasi dovunque. Del che, se volessimo ricercare una causa, dovremmo forse vederla nel fatto che le vespe sogliono nascondere tra le foglie o nella scorza di un albero o sotto terra la loro abitazione, sicché questa non appạre molto spesso davanti agli occhi del popolo con le sue peculiarita che avrebbero dovuto produrre una ricca e varia fioritura di nomi.

\section{Nomi derivati dalla forma:}

1. BUÑA ${ }^{1}$ (v. Meyer-Lübke, REW. 1369): campagna lucch. bugno di vespre com. Vicopisano, Lari bugno ib.

2. Deriv. di Boro ${ }^{2}$ (v. Meyer-Lübke, REW. 1224): Vaud borinda s. f. com.

3. 'buco' + 'pertugio'3: Ancona bugio de vespre' com. fiasca com.

4. Flaska (v. Meyer-Lübke, REW. 3355): Gaiole (Siena)

5. PANIS: bresc. pane dele vespe 5 Melchiori - Meana Sardo (Cagliari) pani espaśa com. - Lanciano (Chieti) pane di vespe ib.

1 Cfr. i nomi dell' alveare in Ape e Alv., pag. 67.

2 Cfr. i nomi dell' alveare, ivi, pag. 61 .

8 Cfr. i nnmi dell' alveare, ivi, pag. 60.

- Accanto a vespraro.

- A Brissago, l'insieme delle cellette del favo è detto i panel (Voc. Sv. It.), a Suna, si dice invece la pangla (ib.). 
6. Torricella Peligna (Chieti) tacca di vespre com. (v. MeyerLübke, $R E W .8534)$.

II. Nomi derivati dalla materia:

BRUSCU (v. Meyer-L., REW. 1342): mil. bruscia ${ }^{1}$ Banfi.

III. Nomi derivati dal contenuto:

A. Derivati da vespa, apis, *tabana, ecc.

I. Derivati da vesPa:

a) -ARIU: rum. vesparie Cihac 312, vespár Puşcariu, arom. yispár ib. - ; Istria vespèra com., Trieste vespèr Kosovitz 502 -; Vanzone (Ossola) vasper Voc. Sv. It., Vogogna (Ossola), Poschiavo vespé ib., Crealla (Canobbio), ${ }^{2}$ Ascona vešpe ib., Palagnedra-Moneto, Caviano, S. Abbondio vespe ib., Gresso veescpei ib., Crana, S. Nazzaro, Cavergnò Bignasco, Maggia, S. Antonio (V. Morobbia), Roveredo, Gandria, Lamone, Breno, Viganello, Torricella, Stabio, Balerna vespee ib., Minusio un vespèe ib., Russo un vaspeij ib., Loco vispei ib., Auressio, Mergoscia, Osco, Rossura, Chironico, Soazza vespei ib., Dalpe vespei ib., Cavigliano, Sementina, Menzonio vešpe ib., Golino vaspę ib., Locarno vispee ib., Brissago vespé ib., Gerra Gamb. vešpee ib., $\mathrm{Cu}-$ gnasco vęspę ib., Campo V. Maggia vešpéra ib., Linescio vašpé ib., Peccia vespé e vaspé ib., Broglio lu vaspee ib., Carasso om vespee ib., Faido, Giornico respei ib., Rivera vespe ib., Rovio, Viggiù, Valsolda vẹspé ib., Riva S. Vitale ul vespée ib., Soragno vešpee ib., Malnate veespé ib., Castasegna vešpär ib., Soglio vaspair ib., Bondo Promontogno al véspir ib., Sondrio vespé ib., mil. vespee ${ }^{3} \mathrm{Banfi}$, bergam. vesper" Tiraboschi, Append. 213, cremon. vespaj Fumagalli; pav. vespè Manfredi, Carpi vesper com., Ravenna, Faenza vispier ${ }^{6}$ ib.; piem. vespé Gavuzzi, Valsesia vespée Tonetti, alta e media Vals. vaspee com., Torino vespe ib., Chieri vespe ib., Ivrea uespe ib., Cuneo vesp ' ib., Tortona vesp $\xi^{\prime}$ ib., Casale Monf. vespd ${ }^{7}$ ib.; genov. vespa, Casaccia, Spezia vespao com. - Oniferi ghespargin ib., Nuoro ghespar ju ib., Sassari vipadyu Atl. C. 789, - Trentir:o, Verona ${ }^{8}$ vespdr corn., Malcesine bespár ib., Vicenza vesparo Nazari, Diz. vic., bell. iesper Nazari, Diz. bell. Motta di Livenza vespar com., Udine g̀espar ib. - Rogliano, Isola, Rossa, Calenzana, Venzolasca bespádyu Atl. C. 789, Nonza, Bastia, San Fiorenzo, Canavaggia, Francardo, Calacuccia, Cervione, Pietraserena, Aleria, Pietrapola, Solenzara, e vespée.

1 Cfr. i nomi dell' alveare in Ape e Alv. pag. 77. Si dice anche bisoeu

2 Dicono anche niada del vessp.

a Accanto a bruscia e bisoeu.

- Accanto a vespere, besper, besbér.

5 Il Gambini (255) scrive vëspe e questa stessa grafia da il Diz. dom. pavese-ilal. Circa il valore di $\ddot{e}$ v. quanto osservai in $A p e$ e Alv. pag. 14, n. 2.

- Accanto a sprer.

7 Accanto a ni do respi.

- Dicono anche grespar o niàl delle vreste. 
Conca, Porto-Vecchio, Sartene, Sotta, Bastelica, Coti-Chiavari vespádyu ib., Belgodere, Calvi, Piedicroce, Galeria, S. Pietro-diVenaco, Pianottoli ųspádyu ib., Corte, Ghisoni vespáyu ib., Asco vespádyu ib., Evisa, Nesa véspadyu(?) ib., Guagno, Cauro vispádyu ib., Bonifacio vęspadjyu ib., Bocognano vęspátyu ib., Piana, Vezzani bispádyu ib., Zicavo, Bicchisano, Levie vispádyu ib., Calcatoggio bespédyu ib., Ajaccio vęspédyu ib. - Aquila, Pescocostanzo, Chieti, Teramo 1 vespara com., Sannio vesparo Nittoli; nap. r'sspara Merlo, 'Fon. del dial. di Sora' in Ann. delle univ. tosc, IV, 186, Sora uaspara ib.; Ugento (Lecce) vesparo com.; Melfi (Potenza) vesparo ib. ; calabr. vesparu Accattatis; sic. vespáji Mortillaro, Graniti, Mandanici, Girgenti, Catania, Trapani visparu com. - Roma, Corneto Tarquinia vesparo ib., Canino, Velletri, 2 Tivoli vespaio ib.; Cagli vesper ib., Macerata Feltria vespraro ib.; Spoleto vesparo ib. Chianni, Volterra, Piancastagnaio, Arezzo vespaio, Lucca e Val di Serchio vespaglio ${ }^{3}$ - ; Fribourg, 4 Valais, Genève voućpi com., Vaud vouepi o guêpière ib., Neuchâtel, Jura Bernois guêpière ib. - a. fr. guespier 5 Rolland XIII, 42, fr. dial. vespie $\mathrm{m}$., béspe $\mathrm{m}$. , bespio $\mathrm{m}$., ghespié m. ib. - prov. vespier Azaïs, delf. guespie, gueipie, Mistral, Tresor II, 107, Var vespie ib., linguad. bespie ib. -; spagn. avispero - port. bespeiro.

Epentesi: retor. vesprèr, ${ }^{6}$ Rabins il višprǵ com., Rhäzüns il vašpr $\dot{\xi}$ ib. - ; Stampa veisprair o vesprair Voc. Sv. It.; Ravenna, Faenza sprer ${ }^{7}$ com., Alfonsine sprer ib., Forlì visprêr ib., Cesena vasprer ib., Terra del Sole, Castrocaro vispré ib.; Sarzana, S. Lazzaro, Castelnuovo Magra vaspraro ib. - abruzz. vesprare Finamore, Casalanguida visprara com. - Fano vesprar ib., Macerata, Ancona 8, Fabriano, Fermo vespraro ib. - Carrara, Codena, Miseglia, Torano, Fontia, Avenza vosprár ib., Sassalbo vošprái ib.

parm. vrespär, Reggio Em. vresper ${ }^{9}$ com., mod. vresper, bologn. verspar 10, Imola vresper com., mirand. ferrar. Bondeno vraspar ib., Argenta vrasper ib. - venez. bresper, Vicenza, Padova bresparo com., Verona grespdr 11 ib., contado veron. vrespdr 12 ib., Nogara brespar ib., poles. bresparo Mazzucchi 30, Rovigo bresparo o vresparo ib. -; fr. dial. grespe m. Rolland XIII, 42.

Ripatransone vesperd com.

1 Dicono anche nito de vespro.

2 Dicono anche gnido de vespe,

3 Accanto a nido di vespre.

- Dicono anche vouepiru e ntd.

- Accanto a guespiere f.

- Accanto a ugnieu da vespras.

- Accanto a vispier.

8 Dicono anche bugio de vespre.

- Accanto a brasca del vrèsp salvadghi, Voc. Regg. I, 126.

10 Accanto a vrespèr o viraspar o vrasper.

$"$ Accanto a vespàr e niall delle vireste.

12 Accanto a niavo de vrèspe. 
b) -ARİA: a fr. guespière f. Rolland XIII, 42, fr. dial. oueppieure f., guêpière f., vouépire f. ib. - Rouergat guespiero, bespiliro s. f. Mistral, Trisor II, 107.

fr. dial. grespere Rolland XIII, 42.

c) -AKīa: Reggio Cal. vesparía com. -; fr. dial. ouaspërî f. Rolland XIII, 42, Vall. waspertye, wasperie f. ib.

d) dial. des Alpes guespatie Mistral II, 107, fr. dial. vespatièyro f. Rolland XIII, 42. Forse da VRSPA + AITA + ARIA.

e) -OLU: Fribourg vouepiru ${ }^{1}$ com.

f) -ONE + ARIU: romagn. vrespuneir com.

2. Deriv. da APIS: Lecce aparu com.

3. Deriv. da *Tabana: Propriano (Corsica) lanádyu Atl. C.789-; lion. taugni (pron. tôgnî) s. m. Puitspelu 401 - Savoie tòni m. Rolland XIII, 42.

4. Deriv. da BIs: mil. bisoeu. ${ }^{2}$

5. Deriv. da Asılus: Mantova asiölèr com. - Sorgà (Verona) asiolir ib.

B. 'Favo': * ${ }_{\text {HRISCA }}$ (v. Meyer-Lübke, REW. 1309): Piacenza brësca com., regg. brasca dél vrèsp salvddghi."

IV. 'Il nido delle vespe':

A. Nivus:

1. retorom. ugnieu da vespras 4 Carisch $180-$; Leontica ni de ninispra Voc. Sv. It., bergam. $\ddot{u} n i d_{e}$ jesbe Tiraboschi, Coccaglio $n i$ de vespe (de martinéi) com.; Casale Monf. $u i d d$ vespi ${ }^{5}$ ib.; Genova nio de vespa ib., Monterosso al Mare, Chiavari nio de respe ib. - sardo niu di espis Porru, Cagliari, Guasila nidu de ispis com., logud. nidu de espes - Pesero (Tirolo) ni di vespe com., Cividale (Udine) nid di ğspis ib., Volpago, Treviso, Mogliano Veneto nio de vespe ib. - Teramo nito de vespró6 ib., Civitelia Alfedena nido di vespre ib.; nap. nizo de le vespe Contursi, Diz. dom., Napoli 1807; S. Severo (Foggia) nito di vespa com., Lecce nido de espe com.; Matera (Potenza) u nïdi du vresplee com. - Velletri gnido di vespe ${ }^{7}$ com. - Lucca e Val di Serchio nido di i'espre ${ }^{8}$ com.

Friburgo nid ${ }^{9}$ com.

1 Accanto a vouepi e nid.

- Accanto a vespee e bruscia.

8 Accanto a vrespèr.

- Accanto a vesprèr.

- Accanto a vespà.

- Accanto a vespara.

- Accanto a vespaio.

8 Accanto a vespagio.

- Accanto a vouépi e vouépiru. 
2. Derivati: Crealla (Canobbio) niada del vešp 1 Voc. Sr: It., Marzio niade de vesp com.

Viggiù niava da vẹšp ${ }^{2}$ Voc. Sv. It.

Contado veron. nidvo de vrispe 3 com.

Verona nial delle vreste 4 com.

B. *rana: Castelfrentano tana di vespre com. - Ozieri tana de espe ib.

C) Deriv. da cubare: Arcola (Genova) cod de vespoe com. V. Voci oscure:

fr. dial. ghýdy' pinère f. Rolland XIII, 42.

1 Accanto a vešpe.

- Accanto a vésspé.

- Accanto a vréspar e bréspiar.

- Accanto a grespàr e vespàr.

Gino Bottiglioni. 